\title{
Antagonismo in vitro de Trichoderma spp. sobre aislamientos de Sclerotinia spp. y Rhizoctonia spp.
}

\section{Antagonism of Trichoderma spp. against isolates of Sclerotinia spp. and Rhizoctonia spp. in vitro}

LILLIANA HOYOS-CARVAJAL 1,4

GLORIA DUQUE 2

SERGIO ORDUZ P ${ }^{3}$
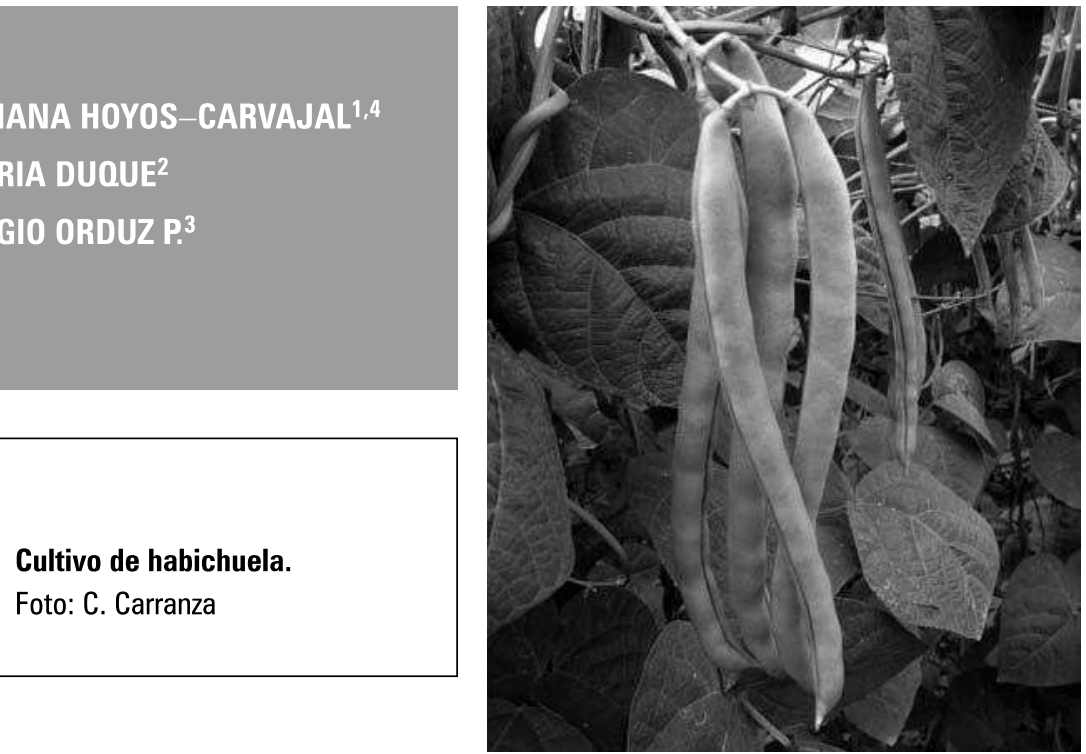

\section{RESUMEN}

El género Trichoderma consta de diversas especies de hongos que tienen la capacidad de parasitar a otros hongos que causan enfermedades en plantas. En el presente estudio, el objetivo fue evaluar la actividad micoparasítica in vitro de 73 aislamientos de Trichoderma spp. sobre tres aislamientos del fitopatógeno Sclerotinia spp. provenientes de habichuela, repollo y pimentón y dos aislamientos de Rhizoctonia provenientes de arroz y de fríjol; se cuantificó evaluando el porcentaje de inhibición del crecimiento micelial o antagonismo siguiendo la metodología de cultivos duales y la calificación de micoparasitismo planteados por Royse y Ries (1978). Para analizar los datos obtenidos en los ensayos, se llevó a cabo la prueba de Kruskall-Wallis, detectando diferencias estadísticas significativas en el grado de parasitismo de aislamientos de Trichoderma spp. sobre los hongos fitopatógenos evaluados. Los aislamientos de Trichoderma con alto grado de micoparasitismo fueron aquellos capaces de combatir a los fitopatógenos en cuatro de las cinco pruebas realizadas, entre los cuales sobresalen T. asperellum T51 y T. harzianum T53. El aislamiento T. harzianum T21demostró tener un grado de antagonismo muy bajo en todas las pruebas realizadas.

\footnotetext{
Departamento de Agronomía, Facultad de Agronomía, Universidad Nacional de Colombia, Bogotá (Colombia).

Colegio Mayor de Antioquia, Medellín (Colombia).

Facultad de Ciencias, Universidad Nacional de Colombia, Medellín (Colombia).

Autor para correspondencia. limhoyosca@unal.edu.co
} 


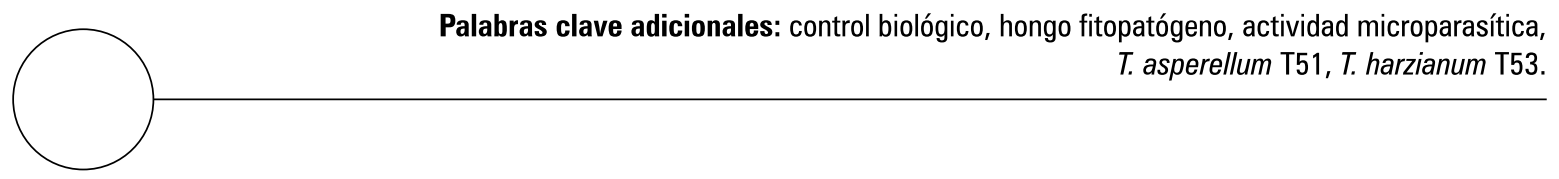

\section{ABSTRACT}

The genus Trichoderma includes various species of fungi that are able to parasitize other fungi, some of which cause plant disease. The goal of the present study was to evaluate, in vitro, the parasitic activity of 73 fungal isolates of Trichoderma spp. on three isolates of the phytopathogens Sclerotinia spp. obtained from green beans, cabbage and pepper, and two isolates of Rhizoctonia spp. obtained from rice and beans. We studied percentage inhibition of mycelial growth or antagonism, using the methodology of dual culture and the classification of mycoparasitism of Royse y Ries (1978). Data was subjected to the Kruskall-Wallis test to detect statistically significant differences in the degree of parasitism of isolates of Trichoderma spp. on the evaluated phytopathogenic fungi. T. asperellum T51 and T. harzianum T53 stood out using the criterion of successful control of the phytopathogens in four of five tests. In contrast, the isolate T. harzianum T21 demonstrated very little antagonism in all tests.

Additional keywords: biological control, phytopathogenic fungi, mycoparasitic activity, T. asperellum T $51, T$. harzianum T 53.

Fecha de recepción: 08-04-2008

Aprobado para publicación: 03-06-2008
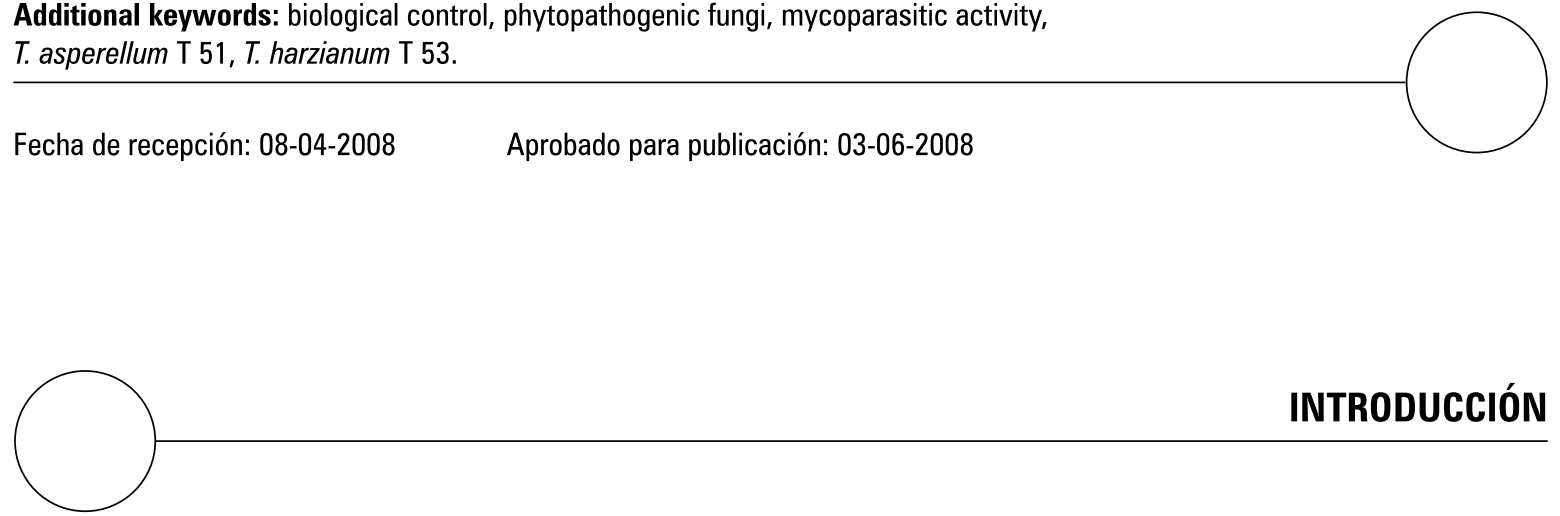

El manejo de enfermedades de plantas ocasionadas por patógenos del suelo son verdaderos retos en la agricultura mundial que por su complejidad requieren un trato más cuidadoso que en el caso de los patógenos de la aerobiota (Castro, 1995). Las enfermedades no sólo tienen el potencial de destruir enteramente las cosechas, aun en los casos en que no causan pérdidas totales, por lo general reducen en forma crónica el rendimiento de la mayoría de los cultivos, obligando a tomar medidas de combate que aumentan los costos de producción y afectan la calidad y la durabilidad de los productos cosechados, de manera que constituyen una de las principales causas de inestabilidad en la empresa agrícola y del déficit alimentario mundial (Strange y Scott, 2005). Más aún aquellas causadas por patógenos formadores de esclerocios, los cuales son agrupaciones compactas de micelio, ricos en materiales de reserva que pueden permanecer en estado de dormancia, hasta 10 años (Schwartz y Pastor-Corrales, 1988). En este grupo de patógenos se encuentran los géneros Rhizoctonia spp. y Sclerotinia spp.

Rhizoctonia solani Khum tiene un micelio incoloro en las primeras etapas de formación y posteriormente se torna amarillo o café cuando madura; consta de células largas con ramificaciones que crecen en ángulo recto con respecto a la hifa principal (Ulloa y Hanlin, 2002). Los síntomas más comunes sobre su hospedero son el ahogamiento de las plántulas y la pudrición de la raíz, 
así como la pudrición y el cáncer del tallo de las plantas adultas y en proceso de crecimiento. Sin embargo, en algunos hospederos también produce pudrición de los órganos vegetales de almacenamiento, así como los tizones o manchas del follaje, especialmente del que se encuentra cerca del suelo (Agrios, 2005). El hongo se disemina principalmente por el agua de riego, por implementos agrícolas y por material de propagación vegetativa (Gilligan et al., 1996).

Sclerotinia sclerotiorum produce hifas blancas en sus estados iniciales; más tarde el micelio se va compactando y toma un aspecto marrón o negro para formar los esclerocios, los cuales son de principal importancia en la supervivencia y epidemiología de Sclerotinia sclerotiorum (Cundom et al., 2002). Los síntomas que produce Sclerotinia varían un poco con el hospedero o la parte afectada de él y con las condiciones del medio ambiente. Las enfermedades causadas por Sclerotinia se conocen bajo una amplia variedad de nombres, siendo las más comunes: la pudrición algodonosa, moho blanco o pudrición blanda aguanosa, pudriciones del tallo y maderamen, marchitez, ahogamiento, pudrición de la corona, tizón de las inflorescencias, nudo rosa, cáncer del tallo, pudriciones de la raíz y corona, entre otras (Agrios, 2005).

En cuanto a métodos de control utilizados para combatir estos hongos, se ha centrado en el uso de agroquímicos, los cuales contribuyen a la acumulación de residuos tóxicos en las cosechas y en el ambiente, con serias consecuencias para la salud humana. Además, los pesticidas no permiten un control efectivo de muchas enfermedades producidas por fitopatógenos del suelo por no ser selectivos. Una alternativa de control ha sido basada en el control biológico, entendido como la reducción de la densidad o de las actividades productoras de enfermedades de un patógeno o parásito, en su estado activo o durmiente, lograda de manera natural o a través de la manipulación del ambiente, del hospedero o de antagonistas del patógeno o plaga que se quiere controlar (Chincholkar y Mukerji, 2007). Esta forma de control de algunos problemas fitosanitarios ha tomado importancia en los últimos años, fundamentada principalmente en la selección de organismos del suelo con propiedades antagónicas sobre otros organismos que producen enfermedades en las plantas (Fravel, 2005), ya que la utilización de microorganismos antagónicos o enmiendas orgánicas se constituyen en una alternativa de control de estos fitopatógenos para mejorar la nutrición y resistencia de las plantas, así como disminuir la incidencia de enfermedades (Nico et al., 2005). El control biológico surge como una alternativa ante la necesidad de reducir los productos químicos, conservando la sanidad de los cultivos. Además, el componente más significativo del suelo que afecta la supervivencia de los esclerocios parece ser el biológico; entre los cuales se encuentran más de 30 especies de hongos y bacterias señaladas como antagonistas (Cundom et al., 2002).

Los antagonistas utilizados para control de enfermedades son generalmente saprófitos, debido a su facilidad de adaptación al medio y a su alta capacidad de competencia por nutrientes frente a otros organismos, a su versatilidad y fácil manipulación. Entre estos se halla el género Trichoderma, que produce tres tipos de propágulos: hifas, clamidosporas y conidios, las cuales son activas contra fitopatógenos en diferentes fases del ciclo de vida, desde la germinación de la espora hasta la esporulación, además ésta reúne una serie de características en su interacción directa con el fitopatógeno, que, según Harman (2000) y Howell (2006), hace de este organismo un buen agente antagonista de hongos fitopatógenos. Entre ellas su capacidad micoparasítica, la cual puede ser definida como el uso del patógeno como alimento por su antagonista, en el que generalmente se ven implicadas enzimas extracelulares como quitinasas, celulasas, $\beta$-1-3-glucanasas y proteasas que lisan o digieren las paredes de los hongos; su capacidad de producción de antibióticos, 
referida a la producción de sustancias tóxicas para otros organismos, las cuales actúan en bajas concentraciones (menores a $10 \mathrm{mg} \mathrm{L}^{-1}$ ); y a su alta competencia por nutrientes y espacio, entendida como el desigual comportamiento de dos o más organismos ante un mismo requerimiento, siempre y cuando la utilización del mismo por uno de los organismos reduzca la cantidad disponible para los demás. Un factor esencial para que exista competencia es que se halla en cantidades insuficientes de un elemento, si hay exceso no hay competencia. La competencia más común es por nutrientes, oxígeno o espacio y, por último, la desactivación de las enzimas de los patógenos (Benítez et al., 2004).

El género Trichoderma es un Ascomycete, perteneciente al orden Hypocreales Mitospóricos, a la familia Hypocreaceae, cuyo estado anamorfo es Trichoderma, y su telomorfo es Hypocrea. Este hongo es cosmopólita en suelo y se halla en madera en descomposición y en material vegetal, las especies de Trichoderma son frecuentes componentes dominantes de la microflora del suelo en una amplia variedad de hábitats, esta capacidad es atribuida al diverso potencial metabólico de las especies de Trichoderma y a su agresiva competencia natural (Samuels, 2006). Trichoderma spp. es bien conocido por la producción de enzimas líticas y la penetración de hifas de éste en el hongo fitopatógeno, tal fenómeno ha sido considerado como la base del antagonismo. Además, se ha demostrado que la interacción de Trichoderma spp. con su huésped es específica y controlada por componentes presentes en la pared celular del fitopatógeno, lo cual hace que estos microorganismos sean reconocidos y posteriormente atacados.

El objetivo de este trabajo fue evaluar la capacidad antagónica de 73 aislamientos de Trichoderma spp de la colección de hongos filamentosos de la Unidad de Biotecnología y Control Biológico de la CIB sobre algunas especies de fitopatógenos tales como Sclerotinia spp. y Rhizoctonia spp.

\section{MATERIALES Y MÉTODOS}

Hongos antagonistas empleados: colección de 73 aislamientos de Trichoderma previamente identificados (Hoyos-Carvajal et al., 2008), pertenecientes a la colección de hongos filamentosos de la Corporación para Investigaciones Biológicas, en Medellín (tabla 1) procedentes de Colombia, Ecuador y Perú, los cuales se cultivaron en medio selectivo para Trichoderma (Elad et al., 1980) y en Agar Papa Dextrosa (PDA), según se requiriera.

Hongos fitopatógenos: colección de hongos fitopatógenos formadores de esclerocios los cuales se encontraban en la Unidad de Biotecnología y Control Biológico de la CIB; Sclerotinia proveniente de habichuela (Phaseolus vulgaris), Sclerotinia proveniente de repollo (Brassica oleracea var. capitata), Sclerotinia proveniente de pimentón (Capsicum spp.), Rhizoctonia proveniente de arroz (Oriza sativa), Rhizoctonia proveniente de fríjol (Phaseolus vulgaris); la cual fue recuperada de plántulas de fríjol, su identificación se realizó de acuerdo con las características descritas por Barnett y Hunter (1998). Estos aislamientos fueron cultivados en PDA (tabla 2).

Pruebas de antagonismo in vitro: cada uno de los aislamientos de Trichoderma spp. y cada fitopatógeno se evaluó según la metodología descrita a continuación. En una caja de Petri con PDA se pone un disco de $5 \mathrm{~mm}$ de diámetro de un cultivo del hongo fitopatógeno durante el tiempo necesario para que este inicie su crecimiento micelial. Transcurrido éste periodo de acondicionamiento, se siembra al lado opuesto, a una distancia de $3 \mathrm{~cm}$, un disco de $5 \mathrm{~mm}$ de diámetro de un cultivo de cada aislamiento de Trichoderma spp. Se prepararon tres réplicas por cada pareja de disco y se dejaron en incubación hasta la toma de datos; bajo condiciones de 25 a $30^{\circ} \mathrm{C}$. Como control, se utiliza un disco de micelio del patógeno sin el antagonista. 


\section{Tabla 1. Aislamientos de Trichoderma spp. probados como antagonistas en sistemas in vitro sobre aislamientos de Sclerotinia spp. y Rhizoctonia spp.}

\begin{tabular}{|c|c|c|c|c|}
\hline Código & Especie & Municipio - Localidad & País & Hábitat \\
\hline CIB T01 & T. koningiopsis & Antioquia, Marinilla & Colombia & Rizósfera de Impatiens \\
\hline CIB T02 & T. harzianum & Antioquia, Marinilla & Colombia & Rizósfera de Cyphomandra betacea \\
\hline CIB T03 & T. harzianum & Antioquia, Carmen de V. & Colombia & Rizósfera de Jasminum \\
\hline CIB T04 & T. asperellum & Tolima, Ibagué & Colombia & Rizósfera de Oriza sativa \\
\hline CIB T05 & T. asperellum & Tolima, Ibagué & Colombia & Rizósfera de Oriza sativa \\
\hline CIB T06 & T. virens & Tolima, Espinal & Colombia & Rizósfera de Oriza sativa \\
\hline CIB T07 & T. harzianum & Tolima, Espinal & Colombia & Rizósfera de Gossypium sp. \\
\hline CIB T08 & T. virens & Santander, Villanueva & Colombia & Rizósfera de Nicotiana tabacum \\
\hline CIB T09 & T. asperellum & Antioquia, Marinilla & Colombia & Rizósfera de Cyphomandra betacea \\
\hline CIB T10 & T. viridescens & Antioquia, Caldas & Colombia & Rizósfera de Impatiens \\
\hline CIB T11 & T. harzianum & Antioquia, Caldas & Colombia & Rizósfera de Impatiens \\
\hline CIB T12 & T. asperellum & Risaralda, Pereira & Colombia & Rizósfera de Morus sp. \\
\hline CIB T13 & T. longibrachiatum & Risaralda, Marsella & Colombia & Rizósfera de Musaceae \\
\hline CIB T14 & T. longibrachiatum & Risaralda, Marsella & Colombia & Rizósfera de Musaceae \\
\hline CIB T15 & T. harzianum & Antioquia, Marinilla & Colombia & Rizósfera de Impatiens \\
\hline CIB T16 & T. harzianum & Región incierta & Colombia & Suelo \\
\hline CIB T17 & T. harzianum & Región incierta & Colombia & Suelo \\
\hline CIB T18 & T. longibrachiatum & Antioquia, Caldas & Colombia & Rizósfera de Guadua angustifolia \\
\hline CIB T19 & T. asperellum & Región incierta & Colombia & Suelo \\
\hline CIB T21 & T. asperellum & Región incierta & Colombia & Suelo \\
\hline CIB T25 & T. asperellum & Región incierta & Colombia & Suelo \\
\hline CIB T26 & T. asperellum & Región incierta & Colombia & Suelo \\
\hline CIB T28 & T. asperellum & Región incierta & Colombia & Suelo \\
\hline CIB T29 & T. longibrachiatum & Región incierta & Colombia & Suelo \\
\hline CIB T30 & T. asperellum & Región incierta & Colombia & Suelo \\
\hline CIB T31 & T. asperellum & Antioquia, El Peñol & Colombia & Rizósfera de Cyphomandra betacea \\
\hline CIB T32 & T. asperellum & Antioquia, Entrerrios & Colombia & Rizósfera de Impatiens \\
\hline CIB T33 & T. asperellum & Antioquia, Entrerrios & Colombia & Rizósfera de Brassica oleracea \\
\hline CIB T34 & Trichoderma spp. & Chocó, Riosucio & Colombia & Rizósfera de Carica papaya \\
\hline CIB T35 & T. harzianum & Chocó, Riosucio & Colombia & Rizósfera de Zingiber oficinale \\
\hline CIB T36 & T. harzianum & Antioquia, Entrerrios & Colombia & Rizósfera de Cyphomandra betacea \\
\hline CIB T37 & T. brevicompactum & Magdalena, Tayrona & Colombia & Suelo de nido de hormiga arriera \\
\hline CIB T38 & T. asperellum & Antioquia, Medellín & Colombia & Fruto de Persea americana \\
\hline CIB T39 & T. asperellum & Antioquia, Caldas & Colombia & Rizósfera de Coffea \\
\hline CIB T40 & T. harzianum & Antioquia, Caldas & Colombia & Rizósfera de Impatiens \\
\hline CIB T41 & T. harzianum & Antioquia, Caldas & Colombia & Rizósfera de Impatiens \\
\hline
\end{tabular}


Tabla 1. Continuación

\begin{tabular}{|c|c|c|c|c|}
\hline Código & Especie & Municipio - Localidad & País & Hábitat \\
\hline CIB T42 & T. harzianum & Antioquia, Caldas & Colombia & Suelo de jardín \\
\hline CIB T43 & T. harzianum & Antioquia, Marinilla & Colombia & Rizósfera de Impatiens \\
\hline CIB T44 & T. harzianum & Magdalena, Tayrona & Colombia & Hojarasca \\
\hline CIB T45 & T. asperellum & Antioquia, Sabaneta & Colombia & Rizósfera de Citrus sinensis \\
\hline CIB T46 & T. asperellum & Risaralda, Marsella & Colombia & Rizósfera de Musa paradisiaca \\
\hline CIB T47 & T. asperellum & Risaralda, Marsella & Colombia & Rizósfera de Musa paradisiaca \\
\hline CIB T48 & T. asperellum & Antioquia, Caldas & Colombia & Rizósfera de Impatiens \\
\hline CIB T49 & T. harzianum & Antioquia, Caldas & Colombia & Rizósfera de Musa paradisiaca \\
\hline CIB T50 & T. asperellum & Antioquia, Caldas & Colombia & Rizósfera de Musa paradisiaca \\
\hline CIB T51 & T. asperellum & Cotopaxi & Ecuador & Pastizal \\
\hline CIB T52 & T. harzianum & Macchu Picchu & Perú & Pastizal \\
\hline CIB T53 & T. harzianum & Santander, Capitanejo & Colombia & Rizósfera de Nicotiana tabacum \\
\hline CIB T55 & T. harzianum & Santander, Pinchote & Colombia & Rizósfera de Nicotiana tabacum \\
\hline CIB T56 & T. harzianum & Santander, Villanueva & Colombia & Rizósfera de Nicotiana tabacum \\
\hline CIB T57 & T. asperellum & Región incierta & Colombia & Suelo \\
\hline CIB T59 & T. harzianum & Santander, Villanueva & Colombia & Rizósfera de Nicotiana tabacum \\
\hline CIB T60 & T. harzianum & Santander, Pinchote & Colombia & Rizósfera de Nicotiana tabacum \\
\hline CIB T61 & T. harzianum & Santander, Villanueva & Colombia & Rizósfera de Nicotiana tabacum \\
\hline CIB T62 & T. asperellum & Región incierta & Colombia & Suelo \\
\hline CIB T63 & T. harzianum & Santander, Villanueva & Colombia & Rizósfera de Nicotiana tabacum \\
\hline CIB T71 & T. asperellum & Región incierta & Colombia & Suelo \\
\hline CIB T73B & T. brevicompactum & Región incierta & Colombia & Suelo \\
\hline CIB T83 & T. asperellum & Región incierta & Colombia & Suelo \\
\hline CIB T84 & T. asperellum & Región incierta & Colombia & Suelo \\
\hline CIB T98 & T. asperellum & Región incierta & Colombia & Suelo \\
\hline CIB T109 & T. asperellum & Región incierta & Colombia & Suelo \\
\hline CIB T110 & T. asperellum & Región incierta & Colombia & Suelo \\
\hline CIB T113 & T. asperellum & Cesar, San Alberto & Colombia & Rizósfera de Elaeis spp. \\
\hline CIB T114 & T. asperellum & Cesar, San Alberto & Colombia & Rizósfera de Elaeis spp. \\
\hline CIB T115 & T. asperellum & Cesar, San Alberto & Colombia & Rizósfera de Elaeis spp. \\
\hline CIB T116 & T. koningiopsis & Cesar, San Alberto & Colombia & Rizósfera de Elaeis spp. \\
\hline CIB T117 & T. asperellum & Cesar, San Alberto & Colombia & Rizósfera de Elaeis spp. \\
\hline CIB T118 & H. jecorina & Cesar, San Alberto & Colombia & Rizósfera de Elaeis spp. \\
\hline CIB T119 & H. jecorina & Cesar, San Alberto & Colombia & Rizósfera de Elaeis spp. \\
\hline CIB T120 & T. asperellum & Cesar, San Alberto & Colombia & Rizósfera de Elaeis spp. \\
\hline CIB T121 & T. asperellum & Antioquia, Cisneros & Colombia & Rizósfera de Saccharum oficinale \\
\hline CIB T124 & T. asperellum & Antioquia, La Unión & Colombia & Rizósfera de Solanum tuberosum \\
\hline
\end{tabular}


Tabla 2. Aislamientos de Sclerotinia spp. y Rhizoctonia spp. usados para pruebas de antagonismo con Trichoderma spp.

\begin{tabular}{|c|l|l|l|}
\hline Código & \multicolumn{1}{|c|}{ Género } & \multicolumn{1}{|c|}{ Hospedero } & \multicolumn{1}{|c|}{ Localidad } \\
\hline Sp 1 & Sclerotinia spp. & Pimentón (Capsicum annuum var. annuum) & Rionegro, Antioquia \\
\hline Sh 2 & Sclerotinia spp. & Habichuela (Phaseolus vulgaris var.vulgaris) & Santuario, Antioquia \\
\hline Sr 3 & Sclerotinia spp. & Repollo (Brassica oleracea var. capitata) & Santuario, Antioquia \\
\hline Ra 1 & Rhizoctonia spp. & Arroz (Oriza sativa) & Palmira, Cali \\
\hline Rf 2 & Rhizoctonia spp. & Fríjol (Phaseolus vulgaris) & Santuario, Antioquia \\
\hline
\end{tabular}

Lectura de ensayos: en cada ensayo se evalúan los criterios de porcentaje de inhibición del crecimiento micelial del patógeno (Royse y Ries, 1978). Después de sembrar el antagonista en las cajas, la actividad de los aislamientos de Trichoderma se clasifica de acuerdo con el grado basado en una escala de 1 a 5 en la cual 1 es el $100 \%$ de micoparasitismo y 5 es $0 \%$ de micoparasitismo. Un aislamiento se considera antagonista contra el patógeno si el promedio es $\geq 3$. A un tratamiento que obtenga un promedio entre 2 y 3 no se le asigna clasificación.

Análisis estadísticos: para determinar diferencias en el efecto de las cepas de Trichoderma sobre la inhibición del radio micelial del patógeno $(P \leq 0,05)$ se analiza con la prueba de Kruskall-Wallis (estadística no paramétrica) y para detectar diferencias entre tratamientos se hacen gráficos de Box and Wisker plots, en los que se determina cuáles de los tratamientos (aislamientos de Trichoderma) presentan mayor grado de micoparasitismo.

\section{RESULTADOS Y DISCUSIÓN}

Según la técnica descrita por Royse y Ries (1978) para la realización de pruebas de antagonismo, debe haber un periodo de acondicionamiento del hongo fitopatógeno, el cual se ve reflejado en el crecimiento sobre el medio de cultivo y varía con respecto a la tasa de crecimiento de cada fitopa- tógeno; para Sclerotinia $48 \mathrm{~h}$ (los tres aislamientos), para Rhizoctonia de arroz menos de $12 \mathrm{~h}, \mathrm{y}$ para Rhizoctonia de fríjol $24 \mathrm{~h}$.

En los resultados obtenidos de los análisis estadísticos en la prueba de Kruskall-Wallis, un valor de $P \leq 0,05$ indica que en todos los tratamientos se encontraron diferencias significativas, como se muestra en la tabla 3. Según los análisis Box and Wister plots, se encontró que los aislamientos $T$. asperellum T51 y $T$. harzianum T53 resultaron tener antagonismo in vitro sobre cinco de los seis aislamientos de fitopatógenos evaluados. Estos aislamientos antagónicos pertenecen a especies diferentes y proceden de hábitats muy diferentes del que fueron tomados los fitopatógenos. También puede afirmarse que la mayoría de hongos con actividad antagónica específica sobre los diferentes fitopatógenos corresponde a aislamientos de T. asperellum, especie que constituye el $49 \%$ de aislamientos probados, así como T. harzianum, que es el 31,5\%.

En contraste, el aislamiento que demostró tener un grado de antagonismo mas bajo en las pruebas realizadas corresponde a $T$. harzianum $\mathrm{T} 21$, lo que indica que aislamientos de una misma especie de Trichoderma son específicos en su actividad micoparasítica, por tanto, no se puede generalizar al decir que determinadas especies de Trichoderma son buenas antagonistas, ya que esto depende del aislamiento en particular y su capacidad de atacar de forma concreta a cepas 


\begin{tabular}{|c|c|c|c|}
\hline Código & Género & Valor $\mathbf{P}$ & Aislamientos de Trichoderma con mayor grado de micoparasitismo ${ }^{1}$ \\
\hline Sp 1 & Sclerotinia spp. & 0,00 & $\begin{array}{l}\text { T2, T4, T5, T6, T7, T9, T16, T17, T25, T26, T28, Т31, Т32, Т33, Т34, Т35, T36, } \\
\text { T40, T46, T50, T51, T53, T56, T83. }\end{array}$ \\
\hline Sh 2 & Sclerotinia spp. & $169 \times 10^{-12}$ & $\begin{array}{l}\text { T2, T4, Т5, T6, T9, T16, T17, T25, Т26, Т28, Т31, Т32, Т33, Т34, Т35, Т36, Т40, } \\
\text { T46, Т50, T51, T53. }\end{array}$ \\
\hline Sr 3 & Sclerotinia spp. & $3.818 \times 10^{-10}$ & T3, T7. \\
\hline Ra 1 & Rhizoctonia spp. & $4.61 \times 10^{-5}$ & $\begin{array}{l}\text { T3, T4, T6, T7, T10, T11, T12, T14, T17, T19, T25, T26, T28, T32, T33, T34, } \\
\text { T35, T36, T37, T40, T46, T47, T49, T50, T51, T53, T55, T57, T59, T60, T71, } \\
\text { T84, T98, T110, T113, T114, T115, T119, T123. }\end{array}$ \\
\hline Rf 2 & Rhizoctonia spp. & $3.229 \times 10^{-10}$ & $\begin{array}{l}\text { T43, T51, T53, T55, T56, T62, T63, T98, T114, T115, T116, T117, T118, T119, } \\
\text { T123. }\end{array}$ \\
\hline
\end{tabular}

${ }^{1}$ Según escala Royse y Ries (1978).

Aislamientos en negrilla son antagónicos para la mayoría de fitopatógenos evaluados.

determinadas de hongos fitopatógenos. Esto es soportado por numerosos reportes de literatura en los cuales diversas especies de Trichoderma han sido reportadas como antagonistas en hongos formadores de esclerocios y es claro que son atributos de aislamientos particulares, no de especies (Elad et al., 1980; Elad 1983; Hjeljord y Tronsmo, 1998; Howell, 2006).

En cuanto a T. brevicompactum T37, T. koningiopsis y $T$. reseii $\mathrm{T} 118$ y $\mathrm{T} 119$, presentan antagonismo contra un único aislamiento de los fitopatógenos probados. De éstas, T. brevicompactum es una especie recientemente descrita en la literatura (Krauss et al., 2004), que se halla restringida al Caribe y de la cual no existen antecedentes como micoparásito de fitopatógenos.

En este estudio se probaron 73 aislamientos de Trichoderma spp. mediante ensayos in vitro que presentaron grado de micoparasitismo variable, calificado con la escala diseñada para tal efecto (figura 1), en los cuales se observó que la tasa de crecimiento del fitopatógeno no afecta la actividad micoparasítica de las especies de Trichoderma spp., es decir, una invasión temprana del medio de crecimiento por parte del fitopatógeno no impide o compite por crecimiento con Trichoderma, por el contrario, este crecimiento micelial precoz del hongo fitopatógeno puede ser rápidamente colonizado y parasitado por Trichoderma spp. Pero esta observación sólo puede llevarse a cabo excediendo los tiempos de lectura recomendados por los autores de la escala de micoparasitismo (Royse y Ries, 1978), como se observó en los ensayos con los aislamientos de Rhizoctonia spp.

Aunque generalmente las especies de Trichoderma son de crecimiento rápido y en pocas horas pueden cubrir la superficie de la caja de Petri, que un aislamiento sea de crecimiento lento no lo excluye como buen antagonista, ya que según Cundom et al. (2002), dicha característica, la tasa de crecimiento, no es obstáculo para que en tales hongos puedan encontrarse aislamientos de buen potencial antagónico.

Por tanto, las pruebas in vitro para determinar la capacidad antagónica de un microorganismo con respecto a otro no representan necesariamente el grado de antagonismo y de control biológico en condiciones naturales, pero reflejan la capacidad 
y la variabilidad genética del antagonista y la del fitopatógeno de invadir o crecer en una caja de Petri o en condiciones in vitro. Es de anotar que dichas pruebas y la escala planteada permiten básicamente cuantificar competencia por nutrientes en el medio controlado, mas no cuantificar como tal el micoparasitismo o antagonismo; porque en tal caso se podría afirmar que si un aislamiento fitopatogénico tiene una tasa de crecimiento mas rápida que el aislamiento del Trichoderma, entonces éste sería antagonista evaluado como grado 3, 4 y 5 en la escala de Royse y Ries (1978) (tabla 4 y figura 1). Las pruebas de antagonismo in vitro permiten realizar la selección preliminar de

Tabla 4. Escala de micoparasitismo, según Royse y Ries (1978).

\begin{tabular}{|c|c|c|}
\hline Grado & Micoparasitismo & $\begin{array}{l}\text { Porcentaje de cubrimiento } \\
\text { del antagonista de la superficie } \\
\text { del medio }\end{array}$ \\
\hline 1 & $\begin{array}{l}\text { El antagonista cubre en un } 100 \% \text { el micelio } \\
\text { del fitopatógeno. }\end{array}$ & $100 \%$ \\
\hline 2 & $\begin{array}{l}\text { El antagonista cubre en un } 75 \% \text { el micelio } \\
\text { del fitopatógeno. }\end{array}$ & $75 \%$ \\
\hline 3 & $\begin{array}{c}\text { El antagonista cubre en un } 50 \% \text { el micelio } \\
\text { del fitopatógeno. }\end{array}$ & $50 \%$ \\
\hline 4 & $\begin{array}{l}\text { El antagonista cubre en un } 25 \% \text { el micelio } \\
\text { del fitopatógeno. }\end{array}$ & $25 \%$ \\
\hline
\end{tabular}

aislamientos con rápido crecimiento en sistemas controlados, es decir, miden competencia, no así micoparasitismo, para el cual se requieren observaciones microscópicas en las que se vea una interacción directa entre estructuras del antagonista y del fitopatógeno y, en virtud a esto, evaluar la capacidad micoparasítica. Invariablemente, las pruebas de antagonismo in vitro requieren ser corroboradas en condiciones de invernadero y campo, porque no siempre los hongos que actúan como antagonistas bajo condiciones controladas tienen la capacidad potencial de usarse en el control biológico, como se ha demostrado en otros experimentos con aislamientos usados en este estudio (Hoyos-Carvajal et al., 2008). Esto se debe a que el hongo antagonista puede modificar o actuar diferente de acuerdo con las características del suelo y las sucesiones microbianas particulares en sistemas in vivo (Garveba et al., 2004).

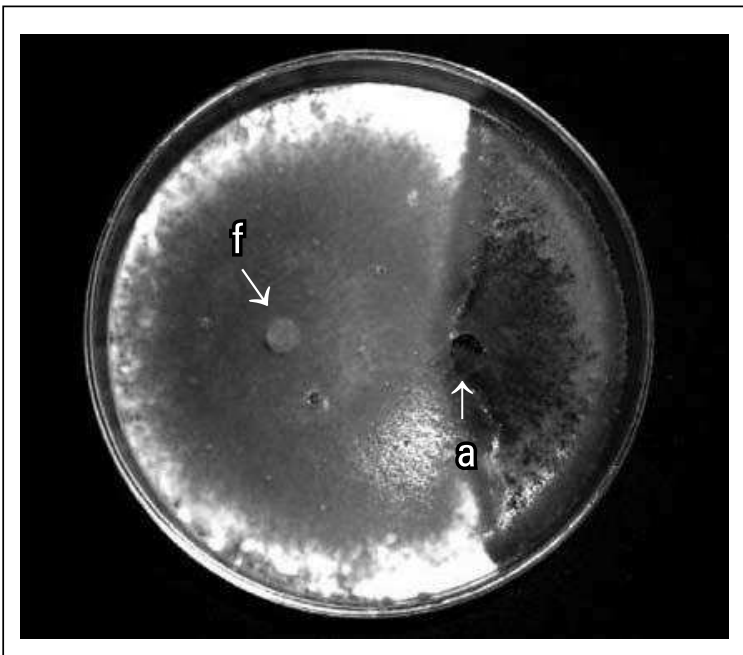

Figura 1. Colonización Trichoderma spp. (a) sobre micelio de Sclerotinia (f). 


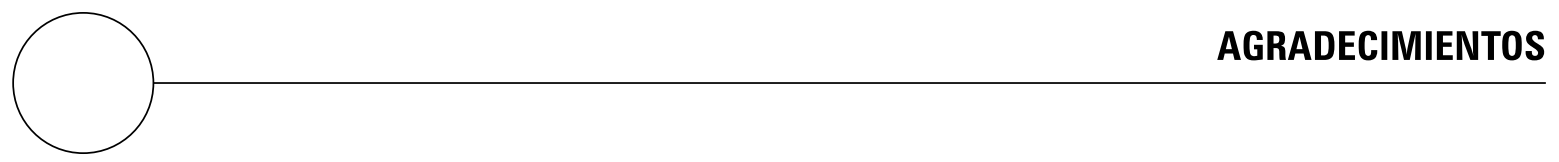

A la Unidad de Biotecnología y Control Biológico de la Corporación para Investigaciones Biológicas (CIP), Medellín, por la financiación de este estudio.

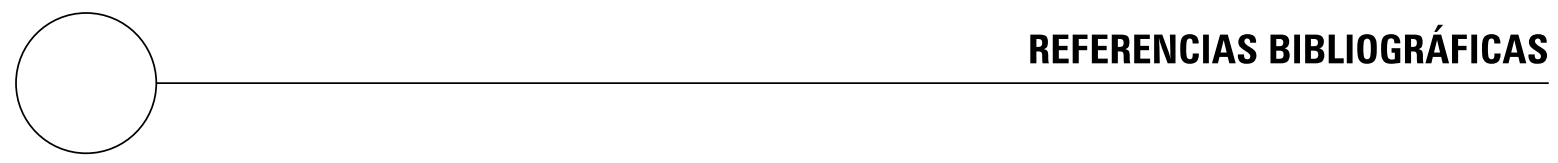

Agrios, G. 2005. Fitopatología. 2a ed. Editorial Limusa, México D.F.

Barnett, H.L. y B.B. Hunter. 1998. Illustred genera of imperfect fungi. $4^{\text {th }}$ ed. American Phytopathological Society, New York, NY.

Benítez, T.; A.M. Rincón; M.C. Limón y A.C. Codón. 2004. Biocontrol mechanisms of Trichoderma strains. Intl. Microbiol. 7(4), 249-260.

Castro, B.L. 1995. Antagonismo de algunos aislamientos de Trichoderma koningii, originados en suelo colombiano contra Rosellinia bunodes, Sclerotinia sclerotium y Pythium ultimum. Fitopatol. Colomb. 19(2), 7-18.

Chincholkar, S. y K.J. Mukerji. 2007. Biological control of plant diseases. Haworth Press, New York, NY.

Cundom, M.A.; S.M. Mazza de Gaiad; M.A. Mazzati de Castañon; S.A. Gutiérrez de Arriola y M. Coutinho. 2002. Actividad antagónica in vitro de Hongos saprófitos sobre Sclerotinia sclerotiorum. En: http:// www.unne.edu.ar/cyt/agrarias/a-037.pdf; consulta: abril de 2008

Elad, Y.; I. Chet y J. Katan. 1980.Trichoderma harzianum: a biocontrol agent effective against Sclerotinia rolfsii and Rhizoctonia solani. Phytopathol. 70(2), 119-121.

Elad, Y.; I.Chet; P. Boyle y Y. Henis. 1983. Parasitismo of Trichoderma spp. on Rhizoctonia solani and Sclerotium rolfsii scanning electron mycroscopy and fluorescence microscopy. Phytopathol. 73, 85-88

Fravel, D.R. 2005. Commercialization and implementation of biocontrol. Annu. Rev. Phytopathol. 43(2), 337-359.
Garveba, P.; J.A. van Veen y J.D. van Elsas. 2004. Microbial diversity in soil: Selección of microbial populations by plant and soil type and implications for disease suppressiveness. Annu. Rev. Phytopathol. 42(2), 243-270

Gilligan, C.A.; S.A. Simons y H.A. Hide. 1996. Inoculum density and spacial pattern of Rhizoctonia solani in field crops of Solanum tuberosum: effects of cropping frequency. Plant Pathol. 45(2), 232-244.

Harman, G. 2000. Myth and dogmas of biocontrol changes in perceptions derived from research on Trichoderma harzianum T22. Plant. Dis. 84(4), 377-393.

Hjeljord, L. y A. Tronsmo. 1998. Trichoderma and Gliocladium in biological control: an overview. pp. 131-151. En: Harman, G.E. y C.P. Kubicek (eds.). Trichoderma and Gliocladium. Vol. 2. Enzymes, biological control and commercial applications. Taylor \& Francis, London.

Hoyos-Carvajal, L.; P. Chaparro; M. Abramsky; I. Chet y S. Orduz. 2008. Evaluación de aislamientos de Trichoderma spp. contra Rhizoctonia solani y Sclerotium rolfsii bajo condiciones in vitro y de invernadero. Agron. Colomb. 26(3), 451-458.

Howell, C.R. 2006. Understanding the mechanisms employed by Trichoderma virens to effect biological control of cotton diseases. Phytopathol. 96(2), 178-180

Kraus, G.F.; I. Druzhinina; W. Gams; J. Bissett; D. Zafari; G. Szakacs; A. Koptchinski; H. Prillinger; R. Zare y C.P. Kubiek. 2004. Trichoderma brevicompactum sp. nov. Mycol. 96(5), 1059-1073.

Nico, A.I.; C.I. Mónaco; G. Dal Bello y H. Alippi. 2005. Efecto de la adición de enmiendas orgánicas al 
suelo sobre la capacidad patogénica de Rhizoctonia solani: ii. Micoflora asociada y antagonismo in vitro de los aislados más frecuentes. Rev. Inv. Agrop. 34(1), 29-44.

Royse, D.J. y S.M. Ries. 1978. The influence of fungi isolated from peach twigs on the pathogenecity of Cytospora cincta. Phytopathol. 68, 603-607

Samuels, G.J. 2006. Trichoderma: Systematics, the sexual state, and ecology. Phytopathol. 96(2), 195-206.
Schwartz, H.F. y M. Pastor-Corrales. 1988. El moho blanco del frijol y su control. Guía de estudio. Centro Internacional de Agricultura Tropical (CIAT), Cali, Colombia.

Strange, R.N. y P.R. Scott. 2005. Plant disease: A threat to global food security. Annu. Rev. Phytopathol. 43(2), 83-116.

Ulloa, M. y R.T. Hanlin. 2000. Illustrated dictionary of mycology. APS Press, St. Paul, MI. 